\title{
$23.5 \%$ efficient monolithic perovskite/organic tandem solar cells based on an ultra-thin metal-like metal-oxide interconnect.
}

Kai Brinkmann ( $\nabla$ brinkmann@uni-wuppertal.de )

University of Wuppertal https://orcid.org/0000-0002-2124-3904

Tim Becker

University of Wuppertal

Florian Zimmermann

University of Wuppertal

Tobias Gahlmann

University of Wuppertal

Manuel Theisen

University of Wuppertal

Tobias Haeger

University of Wuppertal

Selina Olthof

University of Cologne

Cedric Kreusel

University of Wuppertal

Manuel Günster

University of Wuppertal

Timo Maschwitz

University of Wuppertal

Fabian Göbelsmann

University of Wuppertal

Christine Koch

University of Cologne

Dirk Hertel

University of Cologne

Piedro Caprioglio

University of Potsdam

Lorena Perdigon

University of Potsdam

Amran Al-Ashouri 
Helmholtz-Zentrum Berlin

\section{Lena Merten}

Soft Matter Physics, University of Tuebingen

\section{Alexander Hinderhofer}

Universität Tübingen

\section{Frank Schreiber}

University of Tubingen https://orcid.org/0000-0003-3659-6718

\section{Steve Albrecht}

Helmholtz-Zentrum Berlin

\section{Klaus Meerholz}

University of Cologne

\section{Dieter Neher}

University of Potsdam https://orcid.org/0000-0001-6618-8403

\section{Martin Stolterfoht}

University of Potsdam https://orcid.org/0000-0002-4023-2178

\section{Thomas Riedl}

Universitaet Wuppertal

\section{Physical Sciences - Article}

Keywords: Multi-junction solar cells, metal-halide perovskite solar cells, perovskite/organic tandem devices

Posted Date: December 16th, 2020

DOI: https://doi.org/10.21203/rs.3.rs-125381/v1

License: (c) (1) This work is licensed under a Creative Commons Attribution 4.0 International License. Read Full License 


\section{5\% efficient monolithic perovskite/organic tandem solar cells based on an ultra-thin metal-like metal-oxide interconnect.}

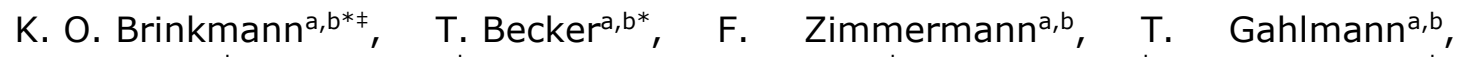
M. Theisen a,b , T. Haeger ${ }^{a, b}$, S. Olthof ${ }^{c}$, C. Kreusela,b, M. Günster ${ }^{a, b}$, T. Maschwitz ${ }^{a, b}$, F. Göbelsmannn, ${ }^{a}$, C. Koch ${ }^{c}$, D. Hertel ${ }^{c}$, P. Caprioglio ${ }^{d}$, L. Perdigond ${ }^{d}$ A. Al-Ashourie, L. Merten ${ }^{f}$, A. Hinderhofer ${ }^{f}$, F. Schreiber ${ }^{f}$, S. Albrechte, K. Meerholz ${ }^{c}$, D. Neher ${ }^{d}$, M. Stolterfoht ${ }^{\mathrm{d}}$ and T. Riedl $\mathrm{l}^{\mathrm{a}, \mathrm{b}} \neq$

a Institute of Electronic Devices, University of Wuppertal, Rainer-Gruenter-Str. 21, 42119 Wuppertal, Germany

${ }^{b}$ Wuppertal Center for Smart Materials \& Systems, University of Wuppertal, RainerGruenter-Str. 21, 42119 Wuppertal, Germany

c Department of Chemistry, University of Cologne, Luxemburger Straße 116, 50939 Cologne, Germany

d Soft Matter Physics, University of Potsdam, Karl-Liebknecht-Str. 24-25, 14476 Potsdam, Germany

e Young Investigator Group - Perovskite Tandem Solar Cells, Helmholtz-Zentrum Berlin, Kekuléstr. 5, 12489 Berlin, Germany

${ }^{f}$ Institute of Applied Physics, University of Tübingen, Auf der Morgenstelle 10, 72076 Tübingen, Germany

${ }^{\ddagger} E$ E-mail: t.riedl@uni-wuppertal.de, brinkmann@uni-wuppertal.de

*equal contribution

Multi-junction solar cells provide an avenue to overcome fundamental efficiency limits of single-junction devices. The facile bandgap tunability of metal-halide perovskite solar cells renders them attractive building-blocks for multi-junction architectures. ${ }^{1}$ Combinations with crystalline silicon and copper indium gallium selenide (CIGS) cells have been reported. ${ }^{2}, 3$ All-perovskite tandem cells have likewise shown promising results. ${ }^{4} 5$ Meanwhile, narrow-gap non-fullerene acceptors (NFA) have revived the area of organic solar cells (OSCs) and unlocked skyrocketing efficiencies. ${ }^{6,7}$ Organic and perovskite semiconductors share similar processing technologies, which renders them attractive partners in multi-junction architectures. As of yet, perovskite/organic tandem cells show subpar efficiencies of $\sim 20$ per cent, limited by the low open circuit 
voltage $\left(V_{o c}\right)$ of wide-gap perovskite cells ${ }^{8}$ and losses introduced by the interconnect between the sub-cells. ${ }^{9,} 10$

Here, we demonstrate two-terminal p-i-n perovskite/organic tandem cells with an efficiency of 23.5 per cent and a high $V_{o c}$ of 2.15 volts, operating near the levels predicted by a semi-empirical model. The perovskite subcells with optimized charge extraction layers afford an unsurpassed combination of a high $\mathbf{V}_{\mathrm{oc}}$ and fill-factor. The organic back-cells provide a high external quantum efficiency in the near-infrared. In surprising contrast to paradigmatic concerns about limited photostability of nonfullerene cells, ${ }^{11}$ we evidence an outstanding operational stability if excitons are predominantly generated on the NFA, which is the case in a tandem cell, where the illumination is spectrally filtered by the perovskite cell. A novel interconnect based on an ultra-thin ( 1.5 nanometers) metal-like indium oxide layer offers unprecedented low optical/electrical losses.

This work sets a new milestone for perovskite/organic tandem devices, that outperform the best $p-i-n$ perovskite single junctions ${ }^{12}$ and are at par with perovskite/CIGS and all-perovskite multi-junctions. ${ }^{13}$ Perovskite/organic tandem architectures bear a realistic potential to reach an efficiency above $31 \%$.

In general, multi-junction solar cells are designed as a series connection of wide-bandgap and narrow-bandgap sub-cells with complementary absorption spectra. An improved overlap with the solar spectrum and reduced thermalization losses are the keys to overcome the ShockleyQueisser efficiency limit of single-junctions. ${ }^{14}$

Hybrid metal-halide perovskites have received tremendous attention as photo-active materials in solar cells. ${ }^{15}$ Their typical $A B X_{3}$ crystal structure comprises methylammonium $\left(\mathrm{MA}^{+}\right)$, formamidinium $\left(\mathrm{FA}^{+}\right)$, or $\mathrm{Cs}^{+}$ions on the $\mathrm{A}$-site, $\mathrm{Pb}^{2+}$ ions on the $\mathrm{B}$-site, and halide ions, such as $\mathrm{I}^{-}$or $\mathrm{Br}^{-}$, on the $\mathrm{X}$-site. Some members of this family, e.g. $\mathrm{FA}_{x} \mathrm{CS}_{1-x} \mathrm{~Pb}\left(\mathrm{I}_{y} \mathrm{Br}_{1-y}\right)_{3}$, afford tunability of the bandgap energy $\left(E_{g}\right)$ between $1.5-2.3 \mathrm{eV}$, mainly by variation of the $\mathrm{I} / \mathrm{Br}$ ratio, ${ }^{16,17}$ rendering them especially attractive for the design of multijunction cells.

All-perovskite tandem cells require narrow-gap materials $\left(E_{\mathrm{g}}<1.3 \mathrm{eV}\right.$ ), where the lead is partially replaced by tin. ${ }^{18,19}$ However, severe stability issues are linked to the unwanted transformation of $\mathrm{Sn}^{2+}$ to $\mathrm{Sn}^{4+}$, which leads to non-intentional p-type doping and an overall degradation of device 
performance. This effect can be slowed down to some extent by additives, such as a surplus of sacrificial $\mathrm{Sn}(0)$ inside the active layer. ${ }^{4}$ Alternatively, c-Si or CIGS back-cells have been employed 2,3 in combination with a wide-gap perovskite front-cell. Some of these tandems already outperform the best c-Si single junctions. ${ }^{2,20}$ On the downside, perovskite/c-Si tandems are not compatible with low-temperature, low-cost, large-area manufacturing - a paradigm that is frequently quoted for perovskite solar technology. In this regard, organic solar cells (OSCs) represent an attractive option as narrow-gap back-cell. OSCs were typically based on so-called bulk-heterojunction architectures, that were formed by a blend of a donor polymer and a fullerene derivative as acceptor. ${ }^{21}$ Owing to the limited tunability of the energy levels of the fullerene, progress in OSCs came to a halt at efficiency levels of about $11 \% .^{22}$ However, with the recent introduction of non-fullerene acceptors (NFAs), OSCs started to see a second wave of outstanding advancement, which has propelled their efficiencies beyond $18 \% .6,7,23$ This progress mainly draws from the narrow energy gap of the acceptor moiety and an efficient exciton dissociation requiring only small energy offsets between donor and acceptor, which is the key to unlock low losses in $V_{\text {oc }}$ compared to $E_{g} / q$ while still providing a high external quantum efficiency (EQE) and thus high short circuit current densities $\left(J_{\mathrm{sc}}\right){ }^{24,25}$

\section{Narrow-gap organic sub-cell}

For the narrow-gap sub-cell in our tandem architecture, we consider an organic photo-active system based on the polymer PM6, that contains a fluorinated-thienyl benzodithiophene, and the NFA Y6, which consists of a ladder-type dithienothiophen[3.2-b]-pyrrolobenzothiadiazole central moiety and 2-(5,6-difluoro-3-oxo-2,3-dihydro-1H-inden-1-ylidene)malononitrile units (Figure 1a). ${ }^{26}$ We employ a $p-i-n$ type device architecture with molybdenum-trioxide as hole extraction layer (HEL) and a bi-layer of $\mathrm{C}_{60} / 2,9$-Dimethyl-4,7-diphenyl-1,10phenanthroline (BCP) for efficient electron extraction. Our cells with binary PM6:Y6 blends (1:1.2) provide a power conversion efficiency (PCE) of up to $16.5 \%$. The EQE spectrum of these devices extends beyond $900 \mathrm{~nm}$ (Figure 1b, Figure S1). Adding a certain concentration of fullerene molecules into the PM6:Y6 photo-active layer to form a so-called ternary system, i.e. PM6:Y6:PC60BM (1:1.2:0.2), improves the blend morphology, which results in enhanced charge transport and reduced non-radiative recombination. 27,28 Thus, a notable boost in cell characteristics is achieved with a PCE up to $17.5 \%$ (for device statistics see Figure S2). We want to 
highlight a notably enhanced EQE of $>85 \%$ for the ternary cells in the range of $\lambda>650 \mathrm{~nm}$, which is the spectral region of operation when combined with a widegap front-cell in a tandem. The absorption spectra of PM6 and Y6 (Figure 1c) show that for $\lambda>650 \mathrm{~nm}$ excitons are predominantly generated on the $Y 6$, which will be shown to be the key that unlocks outstanding device stability.

Stability of NFA cells under continuous operation is still a serious concern and a subject of vigorous scientific research. ${ }^{29}$ To assess the stability of our binary and ternary cells under the illumination conditions applicable in a prospective perovskite/organic tandem cell, we used a filtered white LED (labelled: "LEDvis") and a near infrared (NIR) LED (peak at $850 \mathrm{~nm}$; labelled: "LEDNIR") as light sources, which predominantly create excitons on the PM6 or Y6, respectively (Figure 1c). Under combined VIS/NIR illumination (details can be found in the experimental section), binary and ternary cells show a notable decay of the PCE under continuous operation in the maximum power point (MPP) (Figure 1d), mainly due to a loss in fill factor (FF) (Figure S3). This degradation motif has previously been explained by a photoinduced reorganization in the donor/acceptor blend and the formation of microscopic aggregates of NFA molecules, which leads to a reduced electron mobility and enhanced recombination. ${ }^{30}$ Owing to their improved blend morphology, the decay of the ternary cells is notably slower than that of the binary cells. ${ }^{29}$ However, most strikingly, under NIR illumination, where excitons are solely generated on the $Y 6$, the devices did not show any burn-in and no degradation even under long-term continuous operation for more than 1,000 h. These findings indicate that the detrimental morphological changes, discussed above, would require excitation of the donor polymer PM6 and that they can be substantially mitigated if predominantly the Y6 NFA is excited. On the other hand, we found that upon continuous illumination with the white LED under inert conditions the photoluminescence quantum yield of PM6 shows a notable degradation, while the Y6 is less affected (Figure S4). Therefore, photo-induced degradation of PM6 could likewise play a significant role in addition to possible morphological changes. In any event, our findings contradict the paradigmatic association of non-fullerene solar cells with operational instability, and they present the especially encouraging prospect that the long-term operational stability of perovskite/organic tandem cells will not be limited by the narrow-gap OSC. This is in notable contrast to allperovskite tandems, where the stability issues of Sn-based narrow-gap PSCs are still a very serious issue.

The PM6:Y6 organic system provides an energy-gap of $1.33 \mathrm{eV},{ }^{26}$ and we conducted a semi-empirical electro-optical simulation to identify a suitable wide-gap PSC for a tandem. Details of the simulation can be found in the supporting information. 
According to Figure $1 \mathrm{e}$, an efficiency of $25.5 \%$ is predicted with a perovskite bandgap in the range of 1.85-1.92 eV, assuming a tandem FF of $80 \%$ and a loss in $V_{\text {oc }}$ compared to $E_{g} / q$ of $0.5 \mathrm{~V}$, in each sub-cell. In a more optimistic scenario ( $F F=85 \%$ and loss in $V_{o c}$ compared to $E_{g} / q$ of $0.4 \mathrm{~V}$ ), a tandem of a narrow-gap OSC $\left(E_{g}=1.15 \mathrm{eV}\right)$ with a PSC $\left(E_{g}=1.75 \mathrm{eV}\right)$, provides the prospect to reach an efficiency of $31.3 \%$ (Figure S5). As of yet, efficient OSCs with $E_{g}=1.15 \mathrm{eV}$ have not been developed.
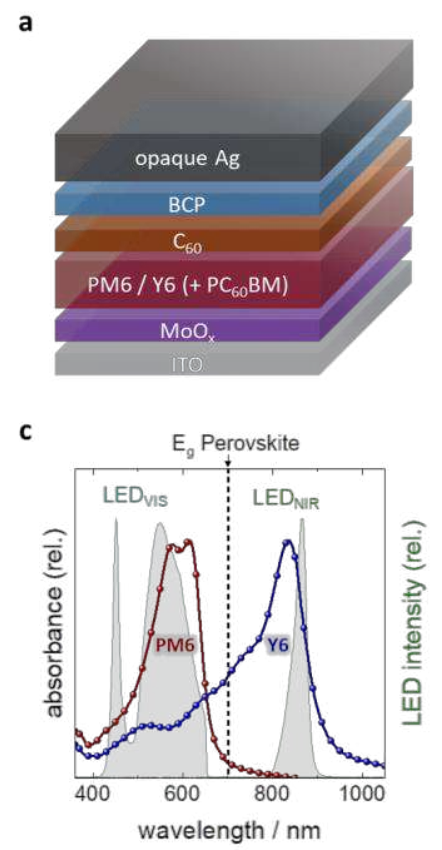

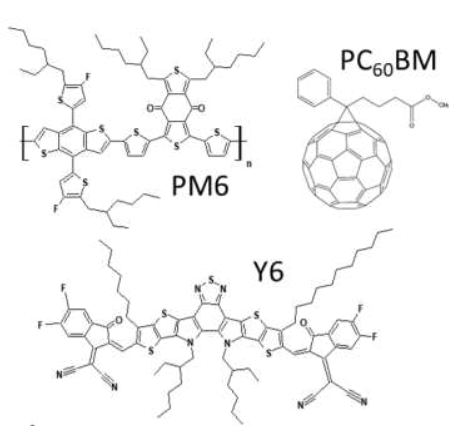

d

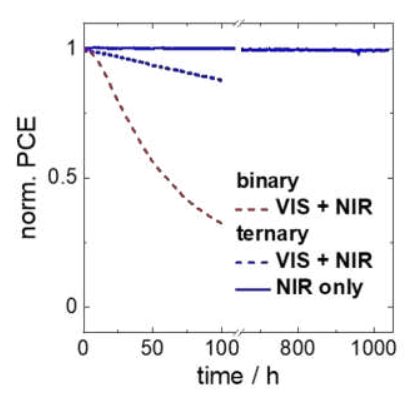

b
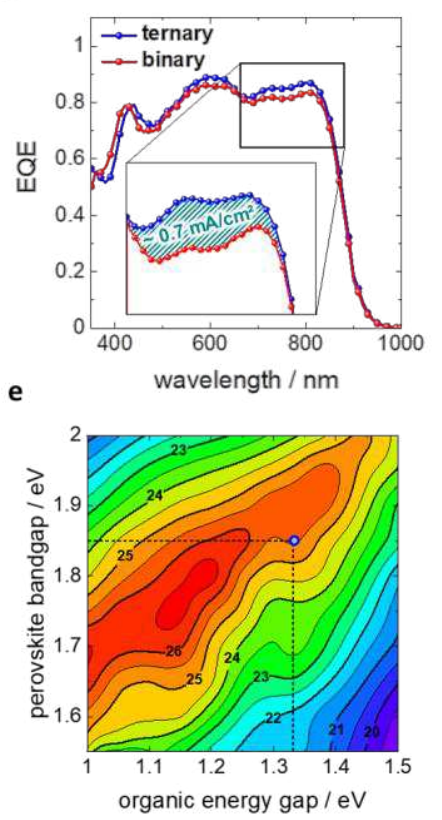

Figure 1 Architecture and properties of the organic sub-cell. a, Layer sequence of the single junction OSC and chemical structure of the molecules used in the photo-active layer. b, External quantum efficiency (EQE) of binary (PM6:Y6) and ternary (PM6:Y6:PC ${ }_{60} \mathrm{BM}$ ) cells with a magnified view of the spectral region $>650 \mathrm{~nm}$, which is the relevant range of operation for the OSC in a perovskite/organic tandem cell. c, absorption spectra of the donor and acceptor molecules used in the photo-active layer. The vertical line marks the $E_{g}$ of a possible wide-gap front cell material. Spectra of (filtered) light emitting diodes used in the stability assessment to selectively excite the donor/acceptor are also shown (labelled "LEDvis" and "LEDNIR"). d, long term stability study of binary and ternary cells continuously operated in the maximum power point (MPP) with exciton generation on both acceptor and donor ("VIS+NIR") or only on the acceptor ("NIR"). Please note the break in the x-axis. e, Realistic semi-empirical model of the tandem cell efficiency vs. energy-gap of organic and perovskite sub-cells. The intersection of the dashed lines marks corresponds to the energy gap of the $\mathrm{PM6}$ :Y6:PC60BM back-cell and the matching energy-gap of the perovskite front-cell. 


\section{Wide-gap perovskite sub-cell}

We selected $\mathrm{FA}_{0.8} \mathrm{CS}_{0.2} \mathrm{~Pb}\left(\mathrm{I}_{0.5} \mathrm{Br} 0.5\right)_{3}$ as suitable perovskite composition with a bandgap of $1.85 \mathrm{eV}$ (Figure S6). Early studies have shown, that for PSCs with $E_{g}>1.75 \mathrm{eV}$, the $V_{\text {oc }}$ did not concomitantly increase with $E_{g}$, which has frequently been attributed to photo-induced halide-segregation in the perovskite into bromine- and iodine-rich domains. ${ }^{31}$ Yet, more recently, recombination losses at the interfaces of the wide-gap perovskite and the adjacent charge extraction layers have been found to be predominately limiting the $V_{o c} .^{8,} 32$ As such, we aimed to minimize these interfacial losses in order to narrow in on the highest possible $V_{o c}$ in our PSCs.

In a first step, we analyzed the quasi-Fermi level splitting (QFLS) in the perovskite upon illumination. The QFLS provides an excellent indication (and upper limit) of the $V_{\text {oc }}$ that can be expected in an actual solar cell and it allows to determine the potential discount in $V_{\text {oc }}$ associated with the respective charge extraction layers adjacent to the perovskite. Poly[bis(4-phenyl)(2,4,6-trimethylphenyl)amine (PTAA) is currently the most prominent hole extraction layer (HEL) in $p-i-n$ PSCs. However, PTAA comes with serious limitations due to the tradeoff between efficient hole transport, which requires the PTAA to be as thin as possible, and selectivity, that has been found to impose a lower limit to the PTAA thickness. ${ }^{33}$ To overcome the issues associated with PTAA, we use [2-(3,6-Dimethoxy-9H-carbazol-9$\mathrm{yl}$ )ethyl]phosphonic Acid (MeO-2PACz) ${ }^{13}$ as $\mathrm{HEL}$, that forms a dense, pinhole-free self-assembled monolayer on the ITO bottom electrode. In a direct comparison perovskite layers on MeO-2PACz as HEL afford a 90 meV larger QFLS compared to their analogues on PTAA (Figure 2a). Interestingly, in stark contrast to PTAA, with MeO-2PACz as HEL, we also did not observe any notable halide segregation under a one sun equivalent illumination on a timescale of several minutes, despite using a Br: I ratio of 0.5:0.5 (Figure S7). This is rather unexpected, as literature suggests notable halide segregation for $\mathrm{Br}$ concentrations in this range. ${ }^{34}$ Our findings demonstrate that the proper choice of HEL allows to mitigate halide segregation in perovskites even with elevated $\mathrm{Br}$-concentrations.

Before studying the impact of the electron extraction layer (EEL), we implemented passivation strategies, such as the addition of excess lead ${ }^{35}$ or the modification of the perovskite surface by the organic halide salt phenethylammonium iodide (PEAI) ${ }^{36}$ which promotes the formation of a 2 -dimensional perovskite capping layer (see Figure S8 - S10). As evidenced by photoelectron spectroscopy, the insertion of $\mathrm{PEA}^{+}$leads to some notable lowering of the perovskite valence band maximum 
(Figure S11), which prevents photo-generated holes from reaching the EEL and thereby improves the selective extraction of electrons.

In the absence of an EEL the surface passivation does not affect the QFLS (Figure 2a), which indicates that defects at the surface or grain boundaries do not impose a limit in this scenario. The situation changes if we complete our $p-i-n$ PSCs by adding $\mathrm{PC}_{60} \mathrm{BM}$ and $\mathrm{Al}$ doped $\mathrm{ZnO}$ nanoparticles (AZO NP) as EEL (Figure 2b). ${ }^{37}$ Note, for the integration of the PSCs in the tandem cells, an additional $\mathrm{SnO}_{x}$ layer, grown by low temperature atomic layer deposition (ALD), is used that serves as permeation barrier, which not only improves the long term stability but also protects the layers underneath against chemical attack by the solvents of subsequent wet chemical processes. ${ }^{38,} 39$

In striking contrast to PTAA, with MeO-2PACz as $\mathrm{HEL}$, the addition of the EEL infers a substantial reduction of the QFLS, and the resulting $V_{\text {oc }}$ shows notable further improvements upon passivation with excess lead and even more by the formation of a 2D perovskite cap (Figure 2c). Ultimately, PSCs with hysteresis-free currentvoltage characteristics are achieved, that provide a very high stabilized $V_{\text {oc }}=1.34 \mathrm{~V}$ (Figure $2 \mathrm{~d}, \mathrm{e}$ ) with a FF that falls within a narrow range of $77-82 \%$ (Figure $2 \mathrm{c}$ and Figure $\mathrm{S} 12$ ). In previous reports, an increase of $V_{\text {oc }}$ typically came at the cost of a lowered $F F$ and a discount in EQE. A comparison with the literature on PSCs with an $E_{g}$ in the range of 1.8-1.9 eV shows that our careful mitigation of losses at the interfaces unlocked access to previously unreached territory of combined high $V_{\text {oc }}$ and FF (Figure 2e). Finally, when operated continuously for more than $100 \mathrm{~h}$, the PCE of the wide-gap PSCs remains above $80 \%$ of its initial value (Figure $2 \mathrm{f}$ ). 

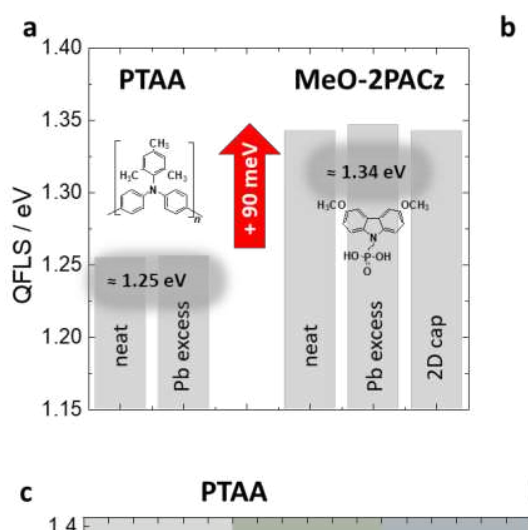

b
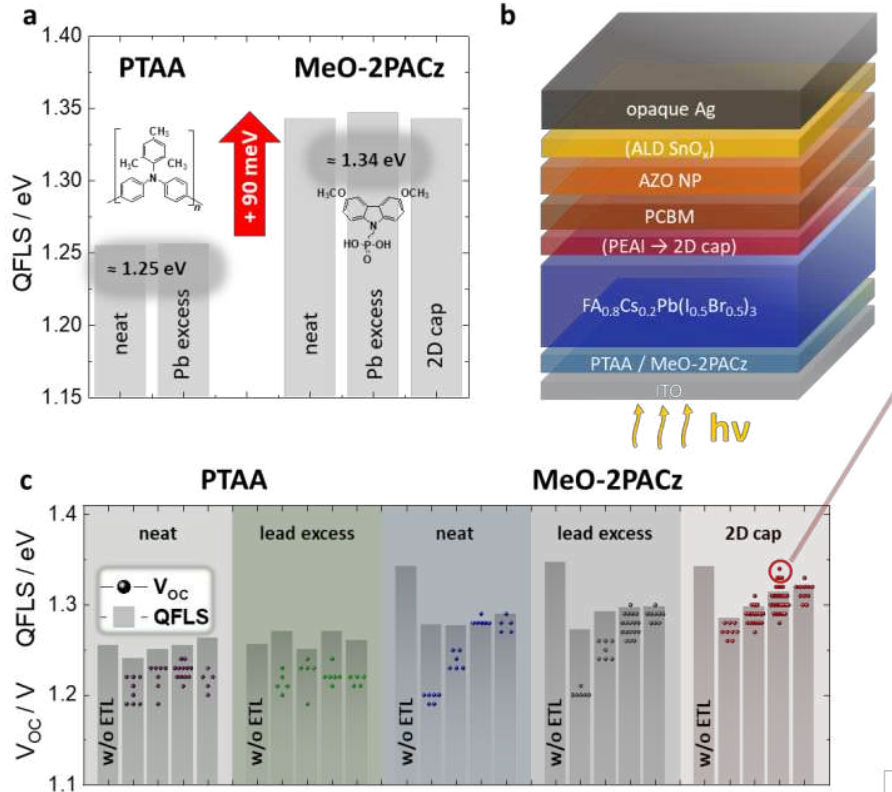

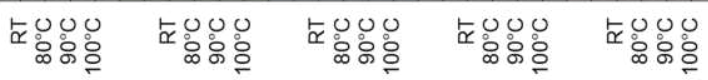

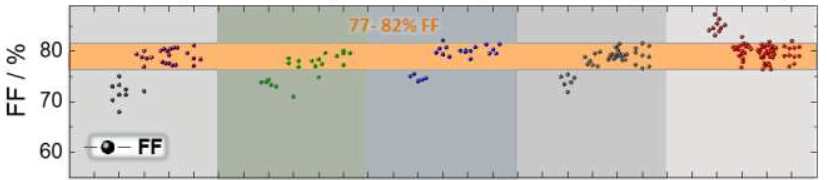

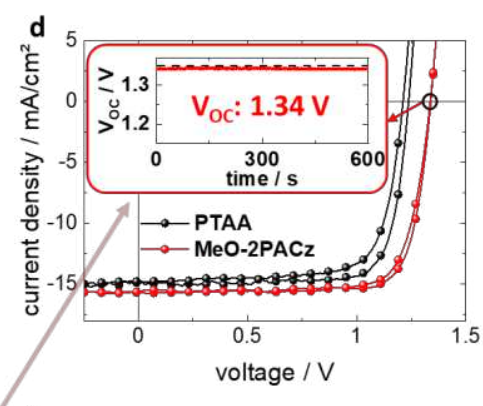

e
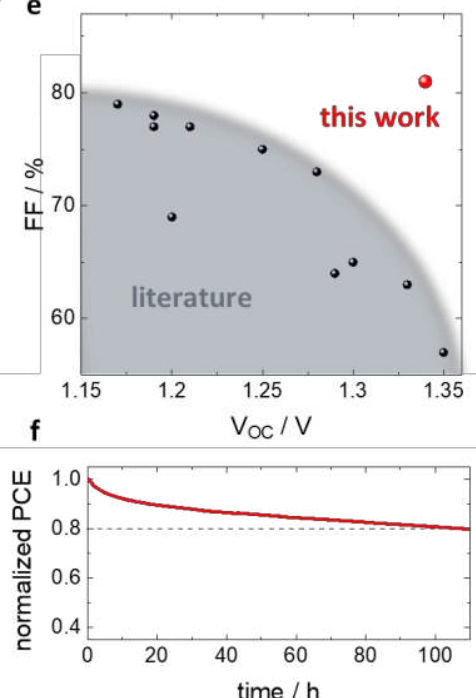

Figure. 2 Optimized wide-gap perovskite sub-cell. a, Splitting of the quasi fermi levels (QFLS) in case of varied hole extraction layers (HEL), i.e. Poly[bis(4phenyl)(2,4,6-trimethylphenyl)amine (PTAA) or [2-(3,6-Dimethoxy-9H-carbazol9-yl)ethyl]phosphonic Acid (MeO-2PACz), and with and w/o usage of excessive lead or a two dimensional capping layer (2D cap, see text) $\mathbf{b}$, Layer sequence of the $p-i-n$ perovskite single junction. $\mathbf{c}$, comparison of the open circuit voltage $\left(V_{o c}\right)$ of actual perovskite solar cells (PSCs) with the corresponding splitting of the quasiFermi level splitting (QFLS) with and w/o electron transport layer (ETL) and varied device annealing temperature. The FF of each cell type is shown in the lower panel. The different temperatures refer to the annealing conditions after deposition of the AZO NP layer. The dots represent the results of individual devices and indicate the experimental spread. d, current density vs. voltage characteristics for champion PSCs with a 2D capping layer (MeO-2PACz SAM as HTL) or $\mathrm{PbI}_{2}$ excess (PTAA as $\mathrm{HTL}$ ) on the perovskite active layer. The inset shows the stabilized $V_{\text {oc }}$ of $1.34 \mathrm{~V}$ for the cell with the SAM as HEL. e, comparison of the $V_{\text {oc }}$ and FF our PSCs with $E_{\mathrm{g}}=1.85 \mathrm{eV}$ to PSCs (with $E_{\mathrm{g}}$ in the range of $1.8-1.9 \mathrm{eV}$ ) reported in the literature. The respective references can be found in Table S1. f, normalized PCE vs. time of the PSCs illuminated with a white LED and operated in the maximum power point. 


\section{Low-loss recombination interconnect}

The interconnect is a key component of monolithic (2-terminal) tandem solar cells (Figure 3a). In a $p-i-n$ tandem, it facilitates the recombination of electrons from the bottom perovskite cell with the holes from the top organic cell, ideally without any loss of $V_{\text {oc }}$ and $F F$. In the simplest approach, one could omit the top electrode of the perovskite cell and stack the organic cell directly on top. In this case, the interface between the low-work-function $\mathrm{SnO}_{x}$ and the high-work-function $\mathrm{MoO}_{x}$ affords only extremely poor, s-shaped $\mathrm{J} / \mathrm{V}$ characteristics of the resulting tandem cell (Figure 3b). This is due to a Schottky barrier of $0.6 \mathrm{eV}$ that forms at the $\mathrm{SnO}_{\mathrm{x}} / \mathrm{MoO}_{\mathrm{x}}$ interface (see Figure $3 \mathrm{c}$, d and Figure $\mathrm{S} 13$ ).

To render the interconnect ohmic, thin layers $(\sim 1 \mathrm{~nm})$ of a metal (Ag or Au) are frequently inserted between top- and bottom-cell. ${ }^{10,}{ }^{40}$ However, even a Ag-layer as thin as $1 \mathrm{~nm}$ already introduces significant optical losses that lower the EQE of the back-cell and the overall $J_{\mathrm{sc}}$ of the tandem cell as shown further below. Thus, we developed an interconnect based on an ultra-thin ALD-grown InOx layer with a thickness of only $\sim 1.5 \mathrm{~nm}$ (details of the ALD growth process can be found in the experimental section). We leverage the unique property of ALD to provide utmost control of layer thickness even on the level of Angströms, which is impossible with conventional deposition techniques. The insertion of $\mathrm{InO}_{x}$ between $\mathrm{SnO}_{x}$ and $\mathrm{MoO}_{x}$ outstandingly improves the $J / V$ characteristics of the tandem cells (Figure $3 \mathrm{~b}$ ). Already 32 ALD cycles (thickness $\sim 1.5 \mathrm{~nm}$, Figure S14) are sufficient to render the $\mathrm{SnO}_{x} / \mathrm{InO}_{x} / \mathrm{MoO}_{x}$ stack ohmic (Figure $3 \mathrm{~b}, \mathrm{c}, \mathrm{d}$ ).

To better understand the interconnect, we conducted an extensive study using photoelectron spectroscopy to determine the energetic line-up (Figure $3 \mathrm{~d}$ and Figure S15-S19). The upward band bending found in case of $\mathrm{SnO}_{x} / \mathrm{MoO}_{\mathrm{x}}$ is completely alleviated by the insertion of the ultra-thin $\mathrm{InO}_{\mathrm{x}}$, enabling barrier-free transport of electrons from the bottom PSC across the $\mathrm{SnO}_{x} / \mathrm{InO}_{x} / \mathrm{MoO}_{x}$ layer sequence. Ultimately, these electrons recombine with the holes from the OSC at the $\mathrm{MoO}_{\mathrm{x}}$ /organic interface. ${ }^{41,42}$ Notably, after completion of a nucleation phase ( 32 ALD cycles), the InOx layer shows a metallic nature with an electron density $\sim 10^{20} \mathrm{~cm}^{-3}$, (Figure 3d,e). As the metallic $\mathrm{InO}_{\mathrm{x}}$ layer is ultra-thin, it still provides a very high sheet resistance $>10^{6} \Omega / \mathrm{sq}$, which is a prerequisite for future largearea scalability as it is of critical importance to avoid shorting of the sub-cells in case of local shunt paths. ${ }^{5}$

High carrier densities typically infer optical absorption, which is another important reason to keep the thickness of the interconnect to a minimum. ${ }^{43}$ Most strikingly, our ultra-thin $\mathrm{InO}_{\mathrm{x}}$ interconnect with a transmittance near unity does not introduce 
notable optical losses (Figure $3 \mathrm{~g}$ ), which boosts the EQE of the organic back-cell and the overall $J_{\mathrm{sc}}$ of the tandem by about $1.5 \mathrm{~mA} / \mathrm{cm}^{2}$ compared to the case of an interconnect based on $1 \mathrm{~nm}$ of $\mathrm{Ag}$, which affords a PCE of only about 20\% (Figure $3 \mathrm{~h}$, Figure S20). ALD allows for large-area, high-throughput processing (even at atmospheric pressure), ${ }^{44}$ and enables conformal coating of textured surfaces that frequently occur in light trapping concepts. ${ }^{2}$ Hence, we foresee that the applicability of our interconnect is not limited to perovskite/organic tandem cells but it may also be favorably used in other tandem cells.
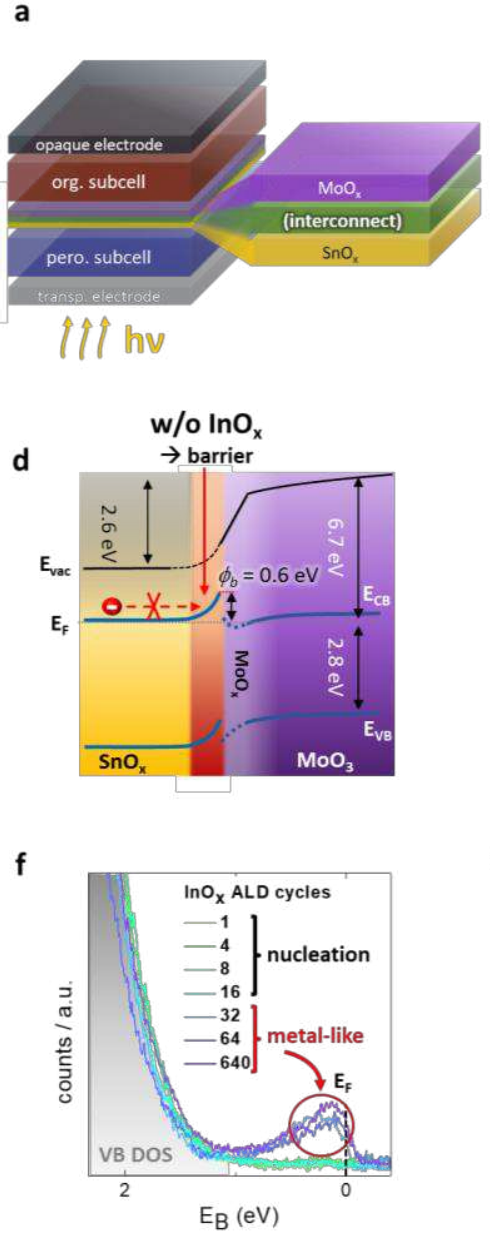
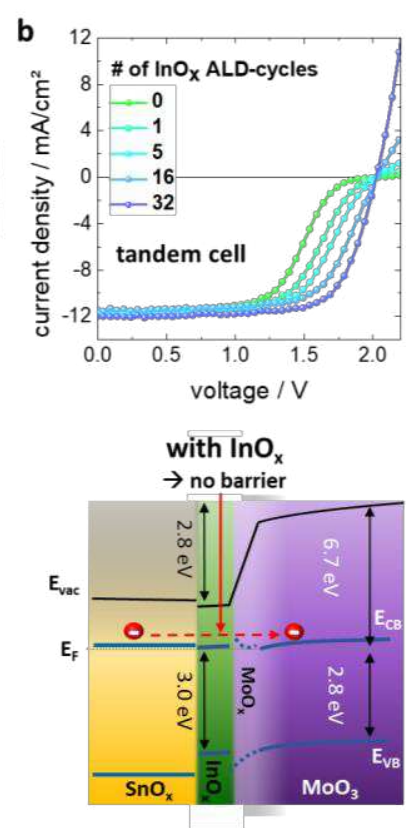

g

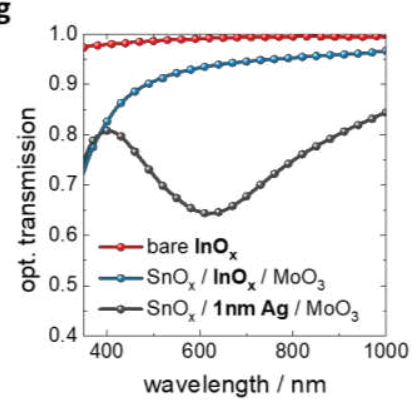

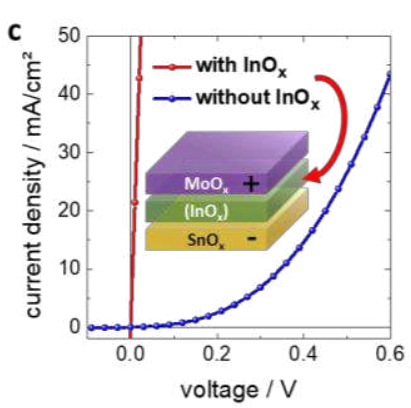
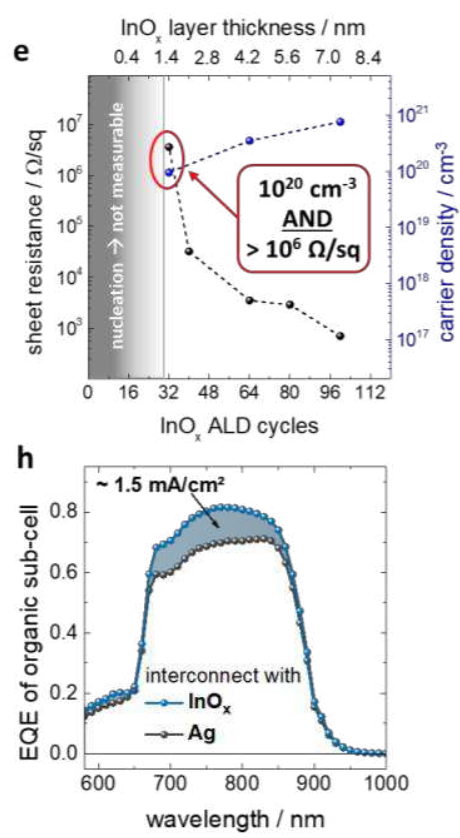

Figure 3 Tandem interconnect. a, Schematic of a perovskite/organic tandem cell with $\mathrm{InO}_{\mathrm{x}}$ or $\mathrm{Ag}$ as interconnect. $\mathbf{b}, \mathrm{J} / \mathrm{V}$ characteristics of tandem cells with varied thickness (\# of ALD cycles) of the $\mathrm{InO}_{x}$ interconnect. $\mathbf{c}_{\boldsymbol{y}} \mathrm{J} / \mathrm{V}$ characteristics of $\mathrm{SnO}_{x} /\left(\mathrm{InO}_{x}\right) / \mathrm{MoO}_{x}$ diodes $\mathbf{d}$, energetic line-up determined with and without $\mathrm{InO}_{x}$. Note, in the first $2 \mathrm{~nm}$ of the molybdenum oxide layer we found a mix of oxidation states of the molybdenum ranging from $\mathrm{Mo}^{2+}$ to $\mathrm{Mo}^{6+}$ (details in Figure S15-S19). The first $2 \mathrm{~nm}$ of the molybdenum oxide are characterized by the presence of $\mathrm{MoO}_{4}{ }^{2-}$ clusters (details in supporting information). e, Sheet resistance and carrier density vs. thickness of the $\mathrm{InO}_{x} . \mathbf{f}$, ultra-violet photoelectron spectroscopy of the 
valence band (VB) density of states (DOS) of $\mathrm{InO}_{\mathrm{x}}$, showing the onset of a metallic behavior at 32 ALD cycles. g, optical transmission of an interconnect based on 32 ALD cycles $(\sim 1.5 \mathrm{~nm})$ of $\mathrm{InO}_{x}$, bare and sandwiched between $\mathrm{SnO}_{x}$ and $\mathrm{MoO}_{\mathrm{x}}$. For comparison, the $\mathrm{InO}_{x}$ has been replaced by a nominally $1 \mathrm{~nm}$ thick layer of $\mathrm{Ag}$. h, resulting EQE spectra of the organic back-cell with $\mathrm{InO}_{x}$ or $\mathrm{Ag}$ as interconnect demonstrating the significant current losses induced by only $1 \mathrm{~nm} \mathrm{Ag}$.

\section{Monolithic perovskite-organic tandem cells}

Drawing from the significant progress outlined above, we prepared monolithic perovskite/organic tandem solar cells with outstanding characteristics. The EQE spectra of the PSC and OSC sub-cells of an optimized perovskite/organic tandem are shown in Figure 4a. Excellent current matching, as required in a series connection, is evidenced by the identical integrated current density of $14.1 \mathrm{~mA} / \mathrm{cm}^{2}$ for both sub-cells. The high $V_{o c}=2.15 \mathrm{~V}$ of the tandem cell results from a perfect addition of the $J / V$ characteristics of the sub-cells without discount in $V_{\text {oc }}$ (Figure $4 \mathrm{~b}$, Figure S21). As a result, a champion tandem cell with a PCE of $23.5 \%$ is achieved (Figure $4 b, c$ ). Our devices state the most efficient monolithic perovskite/organic tandem cells (inset Figure 4c), as of yet, and outperform the most efficient single junction perovskite cells in $p-i-n$ architecture. ${ }^{12}$ The tandem characteristics show a very small statistical variation (Figure S22 \& S23) resulting from a robust processing of the perovskite sub-cell. ${ }^{45}$ We evidence an excellent stability of more than $1,000 \mathrm{~h}$ with no sign of degradation when the devices are kept under inert atmosphere, and $\mathrm{a} \mathrm{T}_{80}$ of $130 \mathrm{~h}$ under continuous operation in the MPP (Figure $4 \mathrm{~d}, \mathrm{e}$ ). Note, the temporal behavior of the tandem under continuous operation is essentially governed by that of the perovskite sub-cell (Figure 2f).

\begin{tabular}{|c|c|c|c|c|c|}
\hline & $\begin{array}{c}\text { scan } \\
\text { direction }\end{array}$ & PCE / \% & FF / \% & Voc / V & $\mathrm{J}_{\mathrm{Sc}} / \mathrm{mA} / \mathrm{cm}^{2}$ \\
\hline \multirow{2}{*}{ PSC } & reverse & 16.8 & 81 & 1.34 & 15.6 \\
\cline { 2 - 6 } & forward & 16.4 & 80 & 1.33 & 15.5 \\
\hline \multirow{2}{*}{ OSC } & invariant & 17.5 & 75 & 0.87 & 26.7 \\
\hline \multirow{2}{*}{ tandem } & reverse & 23.7 & 78 & 2.15 & 14.2 \\
\cline { 2 - 6 } & forward & 23.0 & 76 & 2.12 & 14.2 \\
\hline
\end{tabular}

Table 1: Champion solar cell characteristics of the single junctions and tandem cell derived from their $J / V$ characteristics. Note, for all cells, the $J_{s c}$ 
agrees with the current density obtained from the EQE spectra (deviation less than $1 \%$ ).
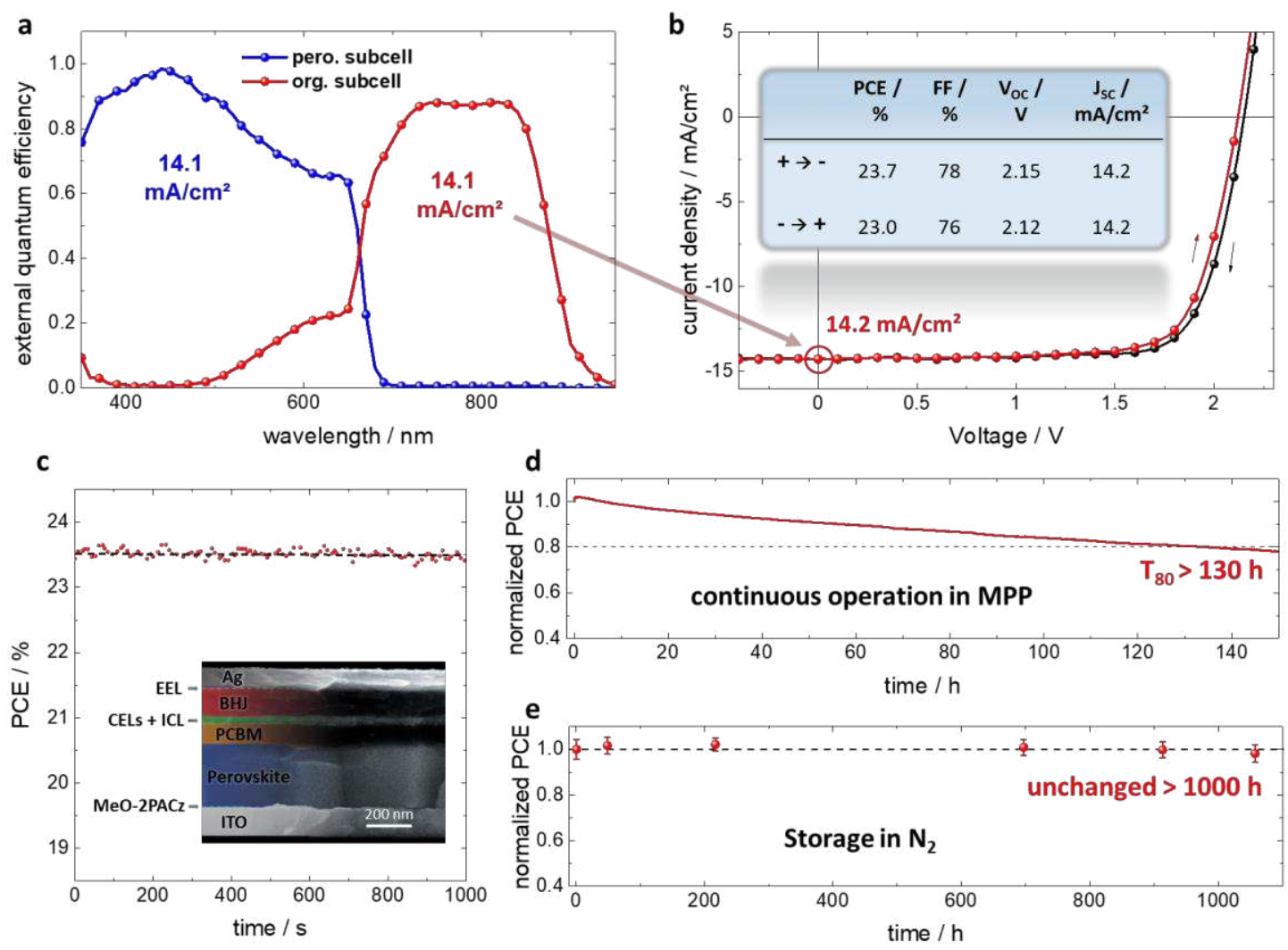

Figure. 4 Perovskite/organic tandem cells. a, External quantum efficiency spectra of both sub-cells in the tandem cell. For reduced reflection, the backside of the substrate was coated with a $100 \mathrm{~nm}$ thick $\mathrm{MgF}_{2}$ layer. $\mathbf{b}, \mathrm{J} / \mathrm{V}$ characteristics of tandem cells. c, stabilized power output (SPO) of the tandem cell. (inset) scanning electron microscopy image of the cell cross section. d, Stability of the tandem cells under continuous operation in the MPP, and $\mathbf{e}$, when stored in $\mathrm{N}_{2}$. 


\section{References}

1. Leijtens T, Bush KA, Prasanna R, McGehee MD. Opportunities and challenges for tandem solar cells using metal halide perovskite semiconductors. Nature Energy 3, 828-838 (2018).

2. Hou $Y$, et al. Efficient tandem solar cells with solution-processed perovskite on textured crystalline silicon. Science 367, 1135-1140 (2020).

3. Han $\mathrm{Q}$, et al. High-performance perovskite/Cu(In, $\mathrm{Ga}) \mathrm{Se}_{2}$ monolithic tandem solar cells. Science 361, 904-908 (2018).

4. Lin R, et al. Monolithic all-perovskite tandem solar cells with $24.8 \%$ efficiency exploiting comproportionation to suppress $\mathrm{Sn}$ (ii) oxidation in precursor ink. Nature Energy 4, 864-873 (2019).

5. Palmstrom AF, et al. Enabling Flexible All-Perovskite Tandem Solar Cells. Joule 3, 2193-2204 (2019).

6. Yan C, et al. Non-fullerene acceptors for organic solar cells. Nature Reviews Materials 3, 18003 (2018).

7. Liu Q, et al. 18\% Efficiency organic solar cells. Science Bulletin 65, 272-275 (2020).

8. Mahesh $\mathrm{S}$, et al. Revealing the origin of voltage loss in mixed-halide perovskite solar cells. Energy \& Environmental Science 13, 258-267 (2020).

9. Li C, Wang Y, Choy WCH. Efficient Interconnection in Perovskite Tandem Solar Cells. Small Methods 4, 2000093 (2020).

10. Chen X, et al. Efficient and Reproducible Monolithic Perovskite/Organic Tandem Solar Cells with Low-Loss Interconnecting Layers. Joule 4, 1594-1606 (2020).

11. Zhan $L$, et al. Over $17 \%$ efficiency ternary organic solar cells enabled by two nonfullerene acceptors working in an alloy-like model. Energy \& Environmental Science 13, 635-645 (2020).

12. Zheng $X$, et al. Managing grains and interfaces via ligand anchoring enables $22.3 \%$ efficiency inverted perovskite solar cells. Nature Energy 5, 131-140 (2020).

13. Al-Ashouri $\mathrm{A}$, et al. Conformal monolayer contacts with lossless interfaces for perovskite single junction and monolithic tandem solar cells. Energy \& Environmental Science 12, 3356-3369 (2019).

14. Shockley W, Queisser HJ. Detailed Balance Limit of Efficiency of p-n Junction Solar Cells. Journal of Applied Physics 32, 510-519 (1961).

15. Graetzel M. The light and shade of perovskite solar cells. Nat Mater 13, 838-842 (2014).

16. McMeekin DP, et al. A mixed-cation lead mixed-halide perovskite absorber for tandem solar cells. Science 351, 151-155 (2016).

17. Tao $\mathrm{S}$, et al. Absolute energy level positions in tin- and lead-based halide perovskites. Nature Communications 10, 2560 (2019).

18. Hao F, Stoumpos CC, Chang RPH, Kanatzidis MG. Anomalous Band Gap Behavior in Mixed $\mathrm{Sn}$ and $\mathrm{Pb}$ Perovskites Enables Broadening of Absorption Spectrum in Solar Cells. J Am Chem Soc 136, 8094-8099 (2014).

19. Zhao $\mathrm{D}$, et al. Efficient two-terminal all-perovskite tandem solar cells enabled by high-quality low-bandgap absorber layers. Nature Energy 3, 1093-1100 (2018). 
20. Green MA, Dunlop ED, Hohl-Ebinger J, Yoshita M, Kopidakis N, Hao X. Solar cell efficiency tables (version 56). Progress in Photovoltaics: Research and Applications 28, 629-638 (2020).

21. Yu G, Gao J, Hummelen JC, Wudl F, Heeger AJ. Polymer Photovoltaic Cells: Enhanced Efficiencies via a Network of Internal Donor-Acceptor Heterojunctions. Science 270, 1789-1791 (1995).

22. Zhao J, et al. Efficient organic solar cells processed from hydrocarbon solvents. Nature Energy 1, 15027 (2016).

23. Cheng P, Li G, Zhan X, Yang Y. Next-generation organic photovoltaics based on nonfullerene acceptors. Nature Photonics 12, 131-142 (2018).

24. Qian $D$, et al. Design rules for minimizing voltage losses in high-efficiency organic solar cells. Nature Materials 17, 703-709 (2018).

25. Liu J, et al. Fast charge separation in a non-fullerene organic solar cell with a small driving force. Nature Energy 1, 16089 (2016).

26. Yuan J, et al. Single-Junction Organic Solar Cell with over 15\% Efficiency Using Fused-Ring Acceptor with Electron-Deficient Core. Joule 3, 1140-1151 (2019).

27. Yu R, Yao H, Cui Y, Hong L, He C, Hou J. Improved Charge Transport and Reduced Nonradiative Energy Loss Enable Over 16\% Efficiency in Ternary Polymer Solar Cells. Advanced Materials 31, 1902302 (2019).

28. Zhu Y, et al. Rational Strategy to Stabilize an Unstable High-Efficiency Binary Nonfullerene Organic Solar Cells with a Third Component. Advanced Energy Materials 9, 1900376 (2019).

29. Gasparini N, et al. Exploiting Ternary Blends for Improved Photostability in HighEfficiency Organic Solar Cells. ACS Energy Letters 5, 1371-1379 (2020).

30. Du X, et al. Unraveling the Microstructure-Related Device Stability for Polymer Solar Cells Based on Nonfullerene Small-Molecular Acceptors. Advanced Materials 32, 1908305 (2020).

31. Slotcavage DJ, Karunadasa HI, McGehee MD. Light-Induced Phase Segregation in Halide-Perovskite Absorbers. ACS Energy Letters 1, 1199-1205 (2016).

32. Peña-Camargo $\mathrm{F}$, et al. Halide Segregation versus Interfacial Recombination in Bromide-Rich Wide-Gap Perovskite Solar Cells. ACS Energy Letters 5, 2728-2736 (2020).

33. Stolterfoht $M$, et al. Approaching the fill factor Shockley-Queisser limit in stable, dopant-free triple cation perovskite solar cells. Energy \& Environmental Science 10, 1530-1539 (2017).

34. Knight AJ, Herz LM. Preventing phase segregation in mixed-halide perovskites: a perspective. Energy \& Environmental Science 13, 2024-2046 (2020).

35. Park B-w, et al. Understanding how excess lead iodide precursor improves halide perovskite solar cell performance. Nature Communications 9, 3301 (2018).

36. Jiang $\mathrm{Q}$, et al. Surface passivation of perovskite film for efficient solar cells. Nature Photonics 13, 460-466 (2019).

37. Brinkmann KO, et al. Suppressed decomposition of organometal halide perovskites by impermeable electron-extraction layers in inverted solar cells. Nature Communications 8, 13938 (2017). 
38. Behrendt A, et al. Highly Robust Transparent and Conductive Gas Diffusion Barriers Based on Tin Oxide. Advanced Materials 27, 5961-5967 (2015).

39. Gahlmann $\mathrm{T}$, et al. Impermeable Charge Transport Layers Enable Aqueous Processing on Top of Perovskite Solar Cells. Advanced Energy Materials 10, 1903897 (2020).

40. Gu S, Lin R, Han Q, Gao Y, Tan H, Zhu J. Tin and Mixed Lead-Tin Halide Perovskite Solar Cells: Progress and their Application in Tandem Solar Cells. Advanced Materials 32, 1907392 (2020).

41. Becker T, et al. All-Oxide MoOx/SnOx Charge Recombination Interconnects for Inverted Organic Tandem Solar Cells. Advanced Energy Materials 8, 1702533 (2018).

42. Meyer J, Hamwi S, Kröger M, Kowalsky W, Riedl T, Kahn A. Transition Metal Oxides for Organic Electronics: Energetics, Device Physics and Applications. Advanced Materials 24, 5408-5427 (2012).

43. Bellingham JR, Phillips WA, Adkins CJ. Intrinsic performance limits in transparent conducting oxides. Journal of Materials Science Letters 11, 263-265 (1992).

44. Hoffmann L, et al. Spatial Atmospheric Pressure Atomic Layer Deposition of Tin Oxide as an Impermeable Electron Extraction Layer for Perovskite Solar Cells with Enhanced Thermal Stability. ACS Applied Materials \& Interfaces 10, 6006-6013 (2018).

45. Brinkmann KO, et al. Extremely Robust Gas-Quenching Deposition of Halide Perovskites on Top of Hydrophobic Hole Transport Materials for Inverted $(p-i-n)$ Solar Cells by Targeting the Precursor Wetting Issue. ACS Applied Materials \& Interfaces 11, $40172-40179$ (2019).

\section{Online Content}

\section{Acknowledgments}

We acknowledge the Deutsche Forschungsgemeinschaft (DFG) (Grant Numbers: RI1551/4-3, RI 1551/12-1 and RI 1551/15-1) and the Bundesministerium für Bildung und Forschung (BMBF) (Grant Number: 01DP20008) for financial support. The research leading to these results has received partial funding from the European Unions's Horizon 2020 Programme under Grant Agreement no. 951774 (FOXES). SO and CK further thank the SCALUP project (SOLAR-ERA.NET Cofund 2, id: 32). We thank Mountain Photonics for providing us with a Prizmatix high-power white LED as well as Tesa Germany for providing us with sealing duct tape.

Additional information

Author contributions

TR, TB and KB conceived and designed the experiments. TB and KB contributed equally to this work. SO and CKo contributed the XPS, UPS and IPES analysis. KB, $\mathrm{TB}, \mathrm{FZ}, \mathrm{CKr}, \mathrm{MG}, \mathrm{TM}$ and $\mathrm{FG}$ were performing the experimental work on the solar cells. TG and MT contributed the metal oxide ALD layers as well as electrical characterization. TH did the cross-section SEM measurements. PC, LP, DN and MS 
designed, conducted and evaluated the PLQY/QFLS studies. AA and SA provided the expertise in the processing of the self-assembled monolayers. $\mathrm{DH}$ and $\mathrm{KM}$ contributed temperature dependent J/V characterization. LM, AH and FS designed and conducted the GIWAXS studies. All authors discussed the results and were involved in the writing.

Supplementary Information available

Competing financial interests: The authors declare no competing financial interests. 


\section{Figures}

a

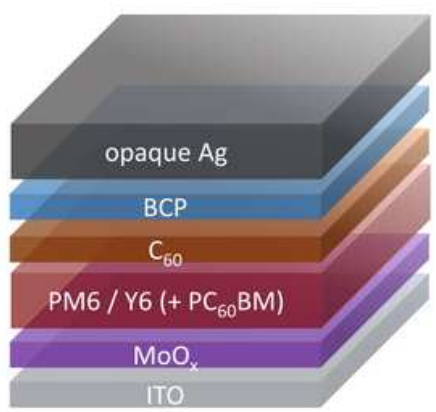

C

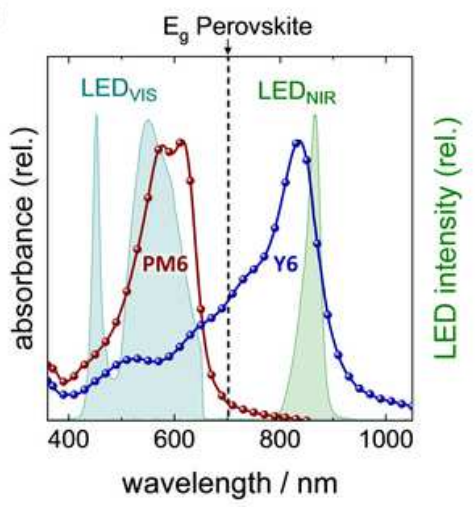

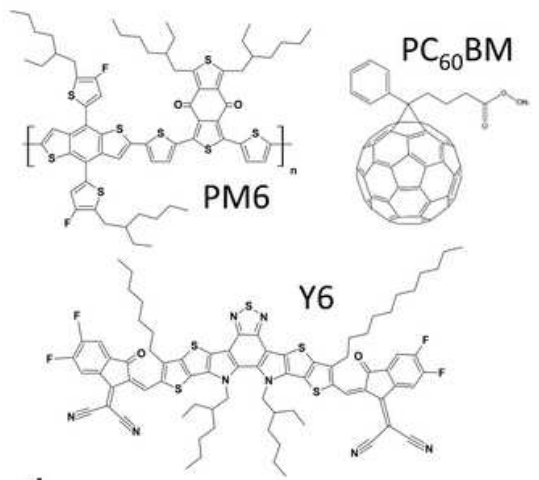

d

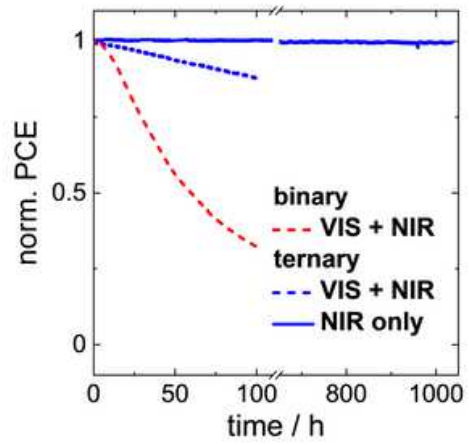

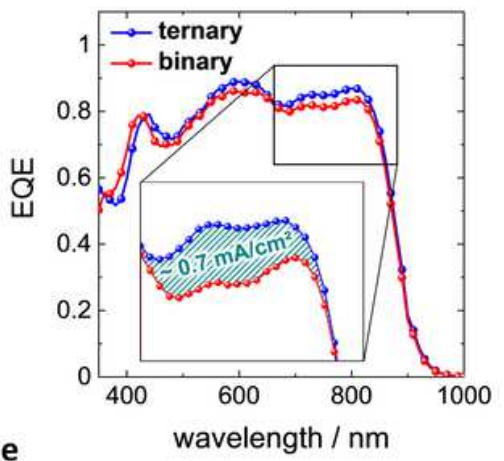

e

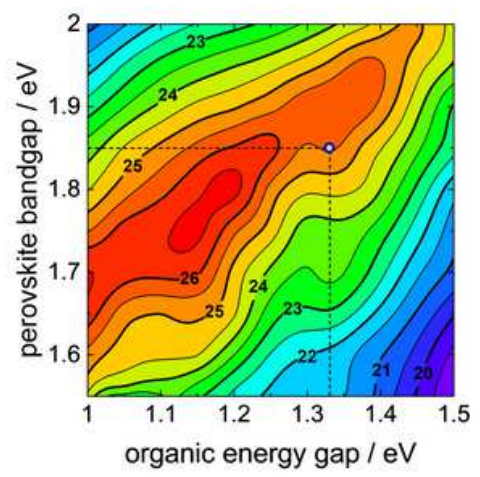

\section{Figure 1}

Architecture and properties of the organic sub-cell. a, Layer sequence of the single junction OSC and chemical structure of the molecules used in the photo-active layer. b, External quantum efficiency (EQE) of binary (PM6:Y6) and ternary (PM6:Y6:PC60BM) cells with a magnified view of the spectral region > $650 \mathrm{~nm}$, which is the relevant range of operation for the OSC in a perovskite/organic tandem cell. C, absorption spectra of the donor and acceptor molecules used in the photo-active layer. The vertical line marks the Eg of a possible wide-gap front cell material. Spectra of (filtered) light emitting diodes used in the stability assessment to selectively excite the donor/acceptor are also shown (labelled "LEDVIS" and "LEDNIR"). d, long term stability study of binary and ternary cells continuously operated in the maximum power point (MPP) with exciton generation on both acceptor and donor ("VIS+NIR") or only on the acceptor ("NIR"). Please note the break in the x-axis. e, Realistic semi-empirical model of the tandem cell efficiency vs. energy-gap of organic and perovskite sub-cells. The intersection of the dashed lines marks corresponds to the energy gap of the PM6:Y6:PC60BM back-cell and the matching energy-gap of the perovskite front-cell. 


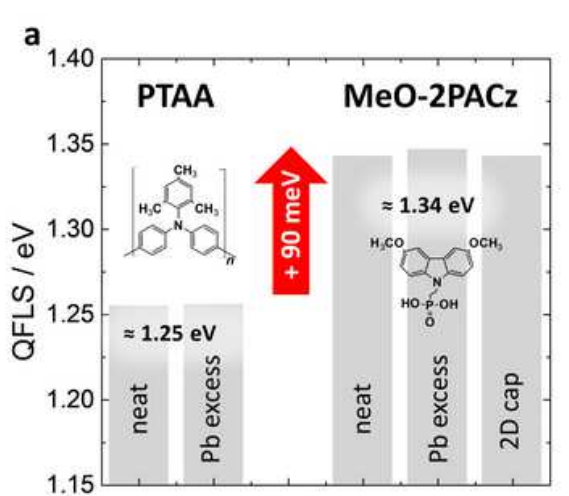

b

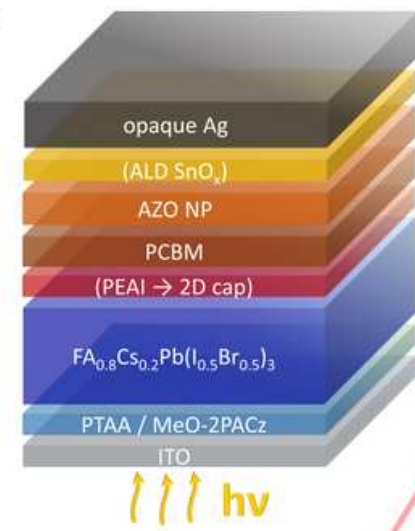

c

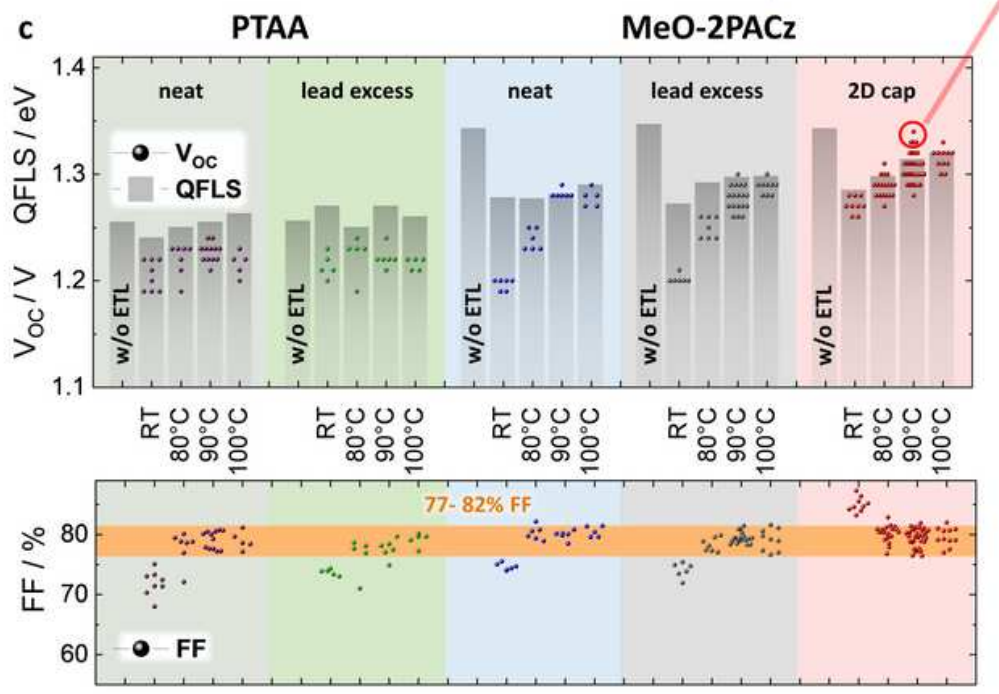

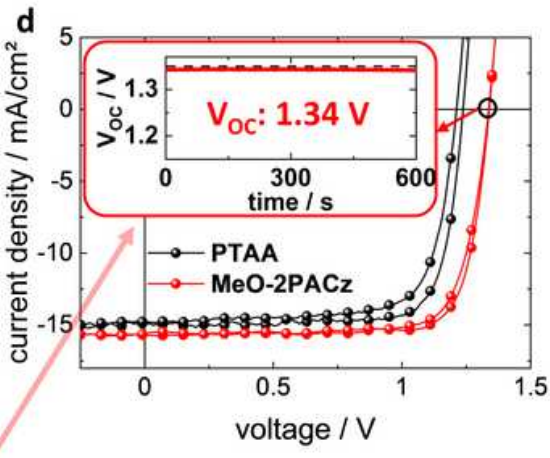
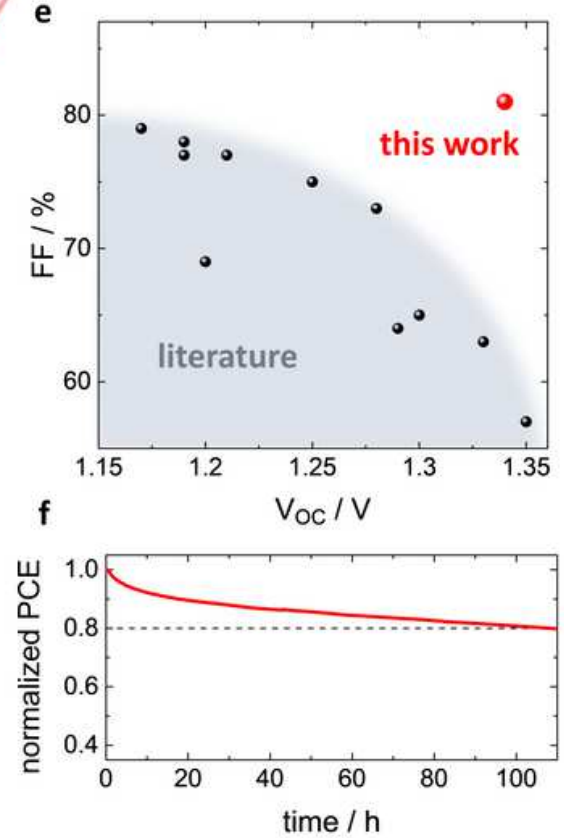

\section{Figure 2}

Optimized wide-gap perovskite sub-cell. a, Splitting of the quasi fermi levels (QFLS) in case of varied hole extraction layers (HEL), i.e. Poly[bis(4- phenyl)(2,4,6-trimethylphenyl)amine (PTAA) or [2-(3,6-Dimethoxy9H-carbazol9-yl)ethyl]phosphonic Acid (MeO-2PACz), and with and w/o usage of excessive lead or a two dimensional capping layer (2D cap, see text) b, Layer sequence of the p-i-n perovskite single junction. $c$, comparison of the open circuit voltage (Voc) of actual perovskite solar cells (PSCs) with the corresponding splitting of the quasiFermi level splitting (QFLS) with and w/o electron transport layer $(E T L)$ and varied device annealing temperature. The FF of each cell type is shown in the lower panel. The different temperatures refer to the annealing conditions after deposition of the AZO NP layer. The dots represent the results of individual devices and indicate the experimental spread. d, current density vs. voltage characteristics for champion PSCs with a 2D capping layer (MeO-2PACz SAM as HTL) or Pbl2 excess (PTAA as HTL) on the perovskite active layer. The inset shows the stabilized Voc of $1.34 \mathrm{~V}$ for the cell with the SAM as HEL. e, comparison of the Voc and FF our PSCs with Eg $=1.85 \mathrm{eV}$ to PSCs (with Eg in the range of 1.8-1.9 eV) reported in the literature. The respective references can be found in Table S1. $f$, 
normalized PCE vs. time of the PSCs illuminated with a white LED and operated in the maximum power point.
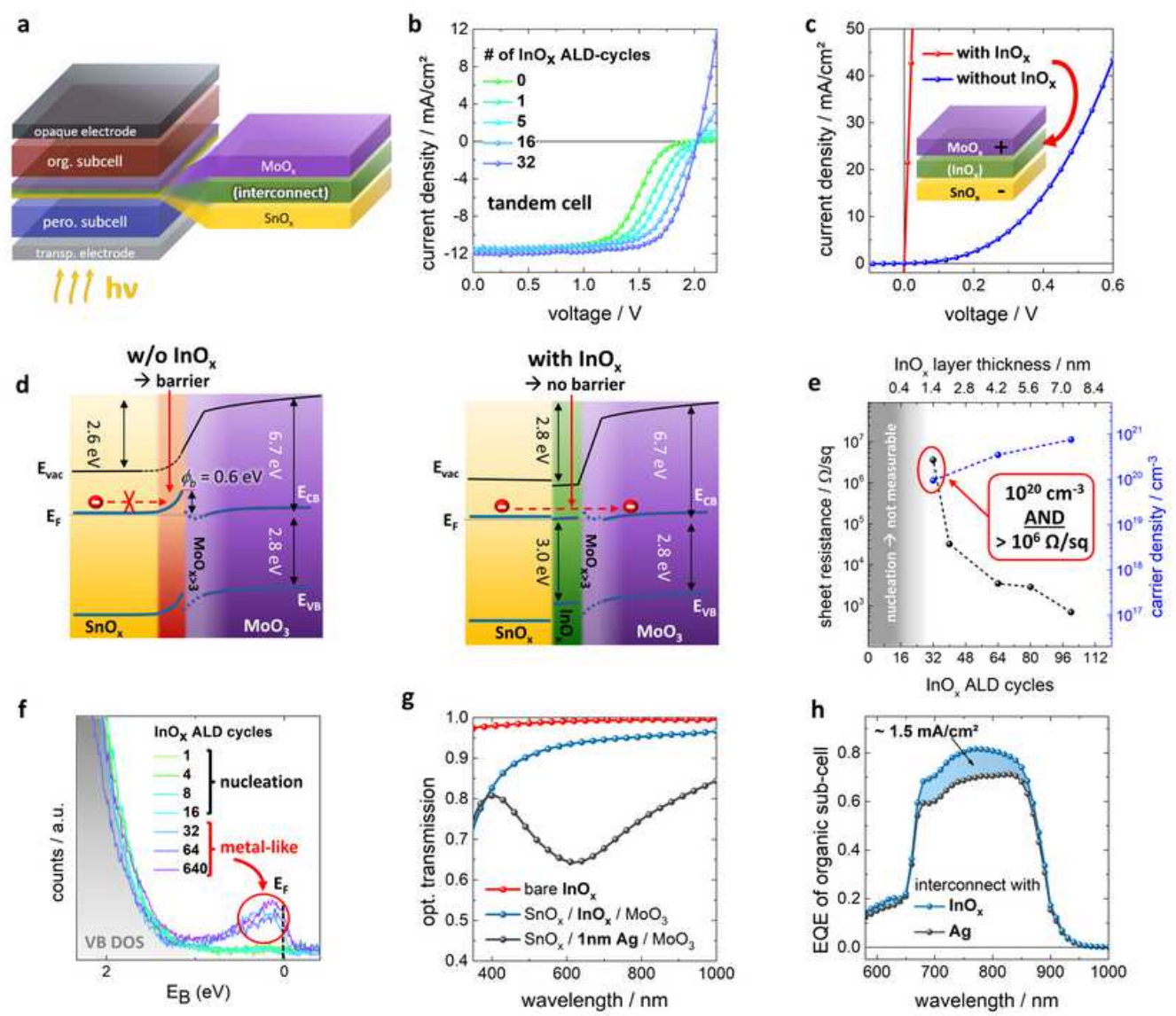

\section{Figure 3}

Tandem interconnect. a, Schematic of a perovskite/organic tandem cell with InOx or Ag as interconnect. b, J/V characteristics of tandem cells with varied thickness (\# of ALD cycles) of the InOx interconnect. c, $\mathrm{J} / \mathrm{V}$ characteristics of $\mathrm{SnOx} /(\operatorname{InOx}) / \mathrm{MoOx}$ diodes d, energetic line-up determined with and without InOx. Note, in the first $2 \mathrm{~nm}$ of the molybdenum oxide layer we found a mix of oxidation states of the molybdenum ranging from Mo2+ to Mo6+ (details in Figure S15-S19). The first $2 \mathrm{~nm}$ of the molybdenum oxide are characterized by the presence of MoO4 2- clusters (details in supporting information). e, Sheet resistance and carrier density vs. thickness of the InOx. $\mathrm{f}$, ultra-violet photoelectron spectroscopy of the 11 valence band (VB) density of states (DOS) of InOx, showing the onset of a metallic behavior at 32 ALD cycles. g, optical transmission of an interconnect based on 32 ALD cycles $(\sim 1.5 \mathrm{~nm})$ of InOx, bare and sandwiched between $\mathrm{SnOx}$ and MoOx. For comparison, the InOx has been replaced by a nominally $1 \mathrm{~nm}$ thick layer of Ag. h, resulting EQE spectra of the organic back-cell with InOx or Ag as interconnect demonstrating the significant current losses induced by only $1 \mathrm{~nm} \mathrm{Ag.}$ 

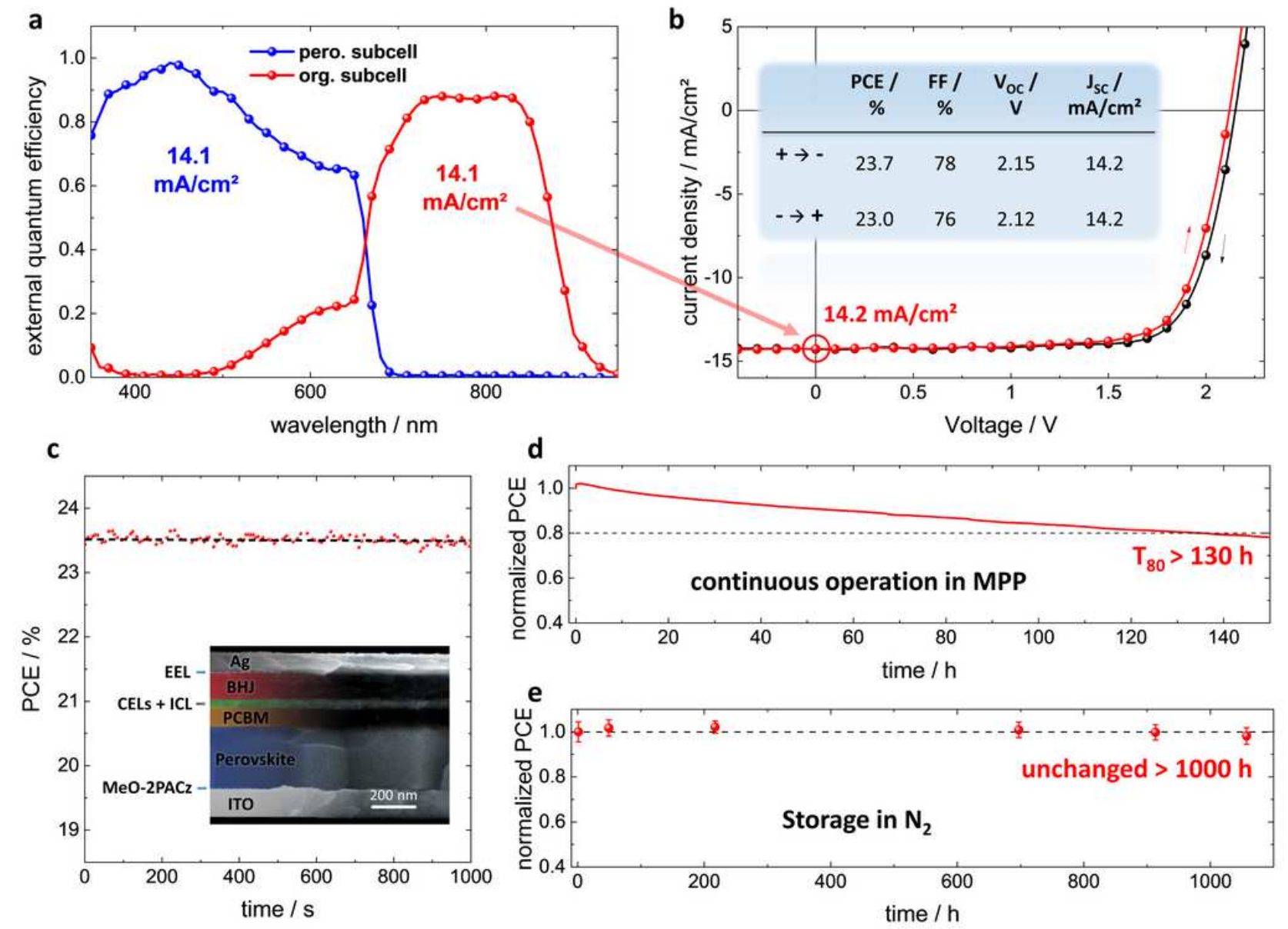

\section{Figure 4}

Perovskite/organic tandem cells. a, External quantum efficiency spectra of both sub-cells in the tandem cell. For reduced reflection, the backside of the substrate was coated with a $100 \mathrm{~nm}$ thick MgF2 layer. b, $\mathrm{J} / \mathrm{V}$ characteristics of tandem cells. c, stabilized power output (SPO) of the tandem cell. (inset) scanning electron microscopy image of the cell cross section. $d$, Stability of the tandem cells under continuous operation in the MPP, and e, when stored in N2.

\section{Supplementary Files}

This is a list of supplementary files associated with this preprint. Click to download.

- PerovskiteOrganikTandemsuppinfoforsubmission2.pdf 